\title{
PERCEPÇÃO DA IMAGEM CORPORAL E ESTADO NUTRICIONAL DE GESTANTES
}

\author{
PERCEPTION OF BODY IMAGE AND NUTRITIONAL STATUS OF PREGNANT \\ WOMEN
}

\section{Fabiane BOCHNIAK'; Daniele Gonçalves VIEIRA²; Gabriela Datsch BENNEMANN³; Camila Daniele FERMINO4; Mariana Abe VICENTE ${ }^{5}$}

\author{
1 - Graduada em Nutrição pela Universidade Estadual do Centro-oeste - UNICENTRO. Brasil. \\ 2 - Docente do Departamento de Nutrição - UNICENTRO, Mestre em Ciências pela Universidade Federal de \\ São Paulo - UNIFESP. Brasil. \\ 3 - Docente do Departamento de Nutrição - UNICENTRO, Mestre em Ciências pela Universidade Federal de \\ Santa Catarina - UFSC. Brasil. \\ 4 - Graduanda em Nutrição pela Universidade Estadual do Centro Oeste - UNICENTRO \\ 5 - Docente do Departamento de Nutrição - UNICENTRO. Mestre em Ciências pela Universidade Federal de \\ São Paulo - UNIFESP. Brasil.
}

Autor para correspondência: marianaav@hotmail.com

\section{RESUMO:}

A gestação é um periodo de grandes modificações no corpo da mulher com consequentente alteração na percepção da imagem corporal. Nesse sentido, o presente estudo tem como objetivo avaliar a percepção da imagem corporal e estado nutricional de gestantes. Para estes foram utilizadas medidas antropometricas de peso e estatura, sendo no periodo gestacional estas medidas aferidas de acordo com Sisvan (2004), no periodo pré gestacional observado dados do Cartão da gestante. Para avaliar a percepção da imagem corporal foi utilizado o questionário Maternal Adjustment and Maternal Attitudes, o qual permite avaliar atitudes e ajustamento materno durante a gestação, contendo uma subescala com questionamentos sobre a avaliação da imagem corporal. Participaram do estudo 26 gestantes, com média de 26,9 semanas no período gestacional. A maioria das gestantes apresentaram eutrofia $11(42,3 \%)$ em ambos os períodos, sendo que no período gestacional houve um aumento no percentual de obesas. Na avaliação da percepção da imagem corporal a questão que demonstrou percepção mais negativa foi: "achou que seu corpo tinha um cheiro agradável?". O estado nutricional das participantes não teve modificações significantes entre os dois períodos, e a percepção da imagem corporal foi mais negativa durante a gestação.

Palavras-chaves: Estado Nutricional, Gestação, Imagem corporal

\section{ABSTRACT:}

Pregnancy is a period of great changes in the female body with consequentente change in perception of body image. In this sense, the present study aims to evaluate the perception of body image and nutritional status of pregnant women. For these anthropometric measures of weight and height were used, the gestational period these measurements were taken according to Sisvan (2004) in pre gestational period-observed data card pregnant. To evaluate the perception of body image questionnaire Maternal Adjustment and Maternal 
Attitude, which allows to evaluate attitudes and maternal adjustment during pregnancy, containing a sub - scale questions about the assessment of body image was used. The study included 26 pregnant women and gestational mean was 26.9 weeks. Most patients were eutrophic 11 (42.3\%) in both periods and during pregnancy there was an increase in the percentage of obese. In assessing the perception of body image issue that showed more negative perception was "thought his body had a pleasant smell?". The nutritional status of the participants had no significant changes between the two periods, and the perception of body image was more negative during pregnancy.

Keywords: Nutritional Status, Pregnancy, Body Image.

\section{INTRODUÇÃO}

O período gestacional corresponde a cerca de 40 semanas, é um momento de transição na vida da mulher, o qual permite o preparo para a maternidade (BRASIL, 2005, RAMOS et al., 2012). Nesse período ocorrem algumas transformações fisiológicas, psicológicas, sociais, físicas e nutricionais, sendo as mais marcantes do ponto de vista nutricional. O aumento no volume dos seios, crescimento da região abdominal, ganho de peso e aumento das necessidades nutricionais (COSTA et al., 2010).

Durante a gestação, há necessidade energética aumentada, devido ao crescimento do feto, da placenta, dos tecidos maternos e para a própria utilização da gestante. No entanto, o aumento da oferta energética torna-se desafiadora ao considerar que o ganho de peso da gestante seja suficiente para o crescimento e desenvolvimento do concepto, sem comprometer o estado nutricional da mãe (SIEGA-RIZ et al., 2009).

Devido ao desenvolvimento fetal, expansão dos tecidos maternos, aumento de líquido extracelular, do volume sanguíneo e a formação do líquido amniótico, o período gestacional é caracterizado pelo aumento do peso corporal, essa a alteração mais característica obsevada a gestação, e que assim o crescimento do feto (ANDRETO et al., 2006; MENEZES, 2004).

O ganho de peso adequado é estimado conforme o estado nutricional prégestacional da mãe, sendo este baseado nas recomendações da Organização Mundial da Saúde (OMS) e o acompanhamento é realizado por meio da Curva de Atalah, 1997, a qual permite a classificação do estado nutricional de gestantes através da relação Índice de Massa Corporal (IMC) atual e idade gestacional. (WHO, 2000).

Em contrapartida a percepção da imagem corporal refere-se a ilustração que a mente tem do corpo, ou seja, o modo como ele se apresenta para o próprio indivíduo (SAIKALI et al., 2004). As mulheres são mais vulneráveis à insatisfação com a imagem corporal, pois esta é gerada pela discrepância entre a percepção e o desejo, em relação a 
forma e tamanho do corpo. Além da estreita relação entre o gênero feminino e a percepção da imagem corporal, verifica-se que durante a gestação essa pode estar mais susceptivel a insatisfação corporal, sendo este um período caracteristico de ganho de peso. (BOSI et al., 2006); COQUEIRO et al., 2008).

A utilização do questionário Maternal Adjustment and Maternal Attitudes (MAMA) elaborado e validado por Kumar, Robson, \& Smith em 1984, permite avaliar atitudes e ajustamento materno durante a gestação, contendo questionamentos pertinentes a avaliação da imagem corporal, e contribuindo assim para detectar situações negativas e positivas ao período gestacional. (FIGUEIREDO et al., 2004).

O estado nutricional adequado, bem como uma percepção positiva da mãe para com sua imagem corporal durante a gestação irá contribuir para um adequado crescimento e desenvolvimento do feto, e para a saúde da gestante.

Nesse sentido o presente estudo tem como objetivo avaliar o estado nutricional e a percepção da imagem corporal de gestantes.

\section{METODOLOGIA}

Estudo transversal realizado com gestantes que frequentavam a Unidade Básica de Saúde (UBS) do município de Boa Ventura de São Roque, Paraná, para receber atendimento no período do pré-natal.

A coleta de dados aconteceu no mês de junho de 2014, após a aprovação do estudo pelo Comitê de Ética em Pesquisa com seres humanos (COMEP) da Universidade Estadual do Centro-Oeste (UNICENTRO) sob o número de protocolo 673.568/2014, sendo que as gestantes foram convidadas a participar da pesquisa no dia em que compareceram na UBS para o acompanhamento pré-natal, as mesmas foram esclarecidas dos procedimentos que seriam realizados. Foram incluídas no estudo todas as gestantes adultas, que compreenderam e concordaram voluntariamente em participar do estudo por meio da assinatura do Termo de Consentimento Livre e Esclarecido (TCLE).

A caracterização da amostra foi composta de um questionário aplicado antes da avaliação antropométrica, contendo questões relacionadas à idade, estado civil, escolaridade e idade gestacional.

A avaliação antropométrica foi realizada por meio das medidas de peso e estatura, aferidas conforme o Sistema de Vigilância Alimentar e Nutricional - SISVAN (ROMEIRO, 2010). A partir do peso e estatura, foi possível calcular o Índice de Massa Corporal (IMC) 
da gestante, por meio da relação peso/ (estatura x estatura) (Kg/m²) (ROMEIRO, A.A.F., 2010). Sendo o resultado obtido utilizado para classificar o estado nutricional das gestantes de acordo com a Curva de Atalah e cols. (ATALAH et al., 1997).

Para classificar o estado nutricional no período pré-gestacional os dados de peso e estatura foram coletados do cartão da gestante, realizado o cálculo do $\mathrm{IMC}\left(\mathrm{Kg} / \mathrm{m}^{2}\right)$, e classificado de acordo com os valores estabelecidos pela Organização Mundial da Saúde (OMS) (WHO, 2000). Embora a OMS estratifique a desnutrição e a obesidade em três categorias, agrupou-se essas classificações para equivaler-se a classificação de Atalah et al, (1997) no período gestacional, ou seja, em baixo peso, eutrofia, sobrepeso e obesidade. (ATALAH et al., 1997; WHO, 2000).

O instrumento utilizado para avaliar a percepção da imagem corporal da gestante em relação ao seu corpo foi o questionário Maternal Adjustment and Maternal Attitudes (MAMA) de auto relato, com 60 itens que se distribuem equitativamente em 5 sub-escalas de 12 itens cada e se reportam às seguintes dimensões: imagem corporal, sintomas somáticos, atitudes para com o companheiro, atitudes perante o sexo, e atitudes para com a gravidez e o bebê. O qual permite tornar mensurável a frequência com que determinadas situações ocorreram, segundo uma escala de tipo Likert que varia entre 1 (Nunca/de forma alguma), 2 (Raramente/muito pouco), 3 (Muito/às vezes) e 4 (Muitíssimo/muitas vezes).

O questionário das subescalas referente a imagem corporal foi aplicado após a avaliação antropométrica, abordando a gestante com questionamentos correspondentes aos períodos da gestação e no mesmo momento foi aplicado questionamentos referente ao período pré-gestacional.

As análises estatísticas foram realizadas com auxílio do Programa Estatístico SPSS versão 20.0. O nível de significância de $5 \%(p<0,05)$ e intervalo de confiança de 95\% foram utilizados. As variáveis quantitativas foram descritas por meio de médias e desvio-padrão, e as variáveis categóricas, qualitativas, foram demonstradas pela frequência absoluta e percentual, utilizando estatística descritiva. Para comparação das médias entre os períodos pré-gestacional e gestacional, foi utilizado o teste-T de Student pareado. Para análise dos dados referente a sub-escala imagem corporal, foi realizada uma média da soma total desta sub-escala, para o período pré-gestacional e período gestacional, sendo levada em consideração a pontuação proposta pelo questionário MAMA (FIGUEIREDO et al., 2004), (Tabela 1), para realizar as correlações foi utilizado a Correlação de Pearson. No estudo os itens que contém o asterisco possuem pontuação invertida, sendo utilizado para a pontuação das variáveis os valores apresentados na tabela, e não a pontuação da resposta 
dada (Tabela 1) (FIGUEIREDO et al., 2004).

TABELA 1. PONTUAÇÃO UTILIZADA PARA CADA ITEM DA SUB-ESCALA IMAGEM CORPORAL DO QUESTIONÁRIO MAMA.

\begin{tabular}{|c|c|c|c|c|}
\hline Descrição & $\begin{array}{l}\text { (Nunca/de } \\
\text { forma alguma) }\end{array}$ & $\begin{array}{l}\text { 2(Raramente/m } \\
\text { uito pouco) }\end{array}$ & 3(Muito/às vezes) & $\begin{array}{l}\text { 4(Muitíssimo/ } \\
\text { muitas vezes) }\end{array}$ \\
\hline Sentiu-se atraente?* & 4 & 3 & 2 & 1 \\
\hline Achou que seu corpo tinha um cheiro agradável?* & 4 & 3 & 2 & 1 \\
\hline Achou que seus seios estavam demasiado pequenos? & 1 & 2 & 3 & 4 \\
\hline Agradou-lhe a forma do seu corpo $?^{*}$ & 4 & 3 & 2 & 1 \\
\hline Achou o seu rosto atraente?* & 4 & 3 & 2 & 1 \\
\hline Achou que seus seios estavam demasiados grandes? & 1 & 2 & 3 & 4 \\
\hline Sentiu orgulho da sua aparência física?* & 4 & 3 & 2 & 1 \\
\hline Sentiu o seu corpo suave e macio?* & 4 & 3 & 2 & 1 \\
\hline Sentiu o seu corpo desajeitado ou sem graça? & 1 & 2 & 3 & 4 \\
\hline Achou a sua constituição física frágil ou débil? & 1 & 2 & 3 & 4 \\
\hline Achou os seus seios atraentes?* & 4 & 3 & 2 & 1 \\
\hline Achou-se muito gorda? & 1 & 2 & 3 & 4 \\
\hline
\end{tabular}

\section{RESULTADOS}

Participaram do estudo 26 gestantes com média de idade $23,7 \pm 5,8$ anos.

A maioria das gestantes 77\% $(n=20)$ viviam em união estável. Quanto à escolaridade $65,4 \%(n=17)$ apresenta 8 anos ou mais de estudo. O período gestacional médio foi $26,9 \pm 8,5$ semanas, sendo que a maioria das participantes estavam no segundo trimestre de gestação 51,6\% (n=16) (Tabela 2).

O estado nutricional das gestantes segundo o IMC, demonstrou que a maioria $42 \%$ $(n=11)$ eram eutróficas em ambos os períodos e que no período gestacional $30,8 \%(n=8)$ das gestantes apresentavam obesidade. No entanto não verificamos diferença significante $(p=0,161)$ no estado nutricional entre os períodos avaliados (Tabela 3 ). 
TABELA 2. CARACTERIZAÇÃO DAS GESTANTES AVALIADAS NA CIDADE DE BOA VENTURA DO SÃO ROQUE-PR NO PERÍODO DE JUNHO DE 2014.

\begin{tabular}{llll}
\hline Parâmetros & & $\mathbf{n}$ & $\%$ \\
\hline Estado civil & Solteira & & 23,1 \\
& Casada & 6 & 46,2 \\
& União estável & 12 & 30,8 \\
& & 8 & \\
Escolaridade & $<$ anos & 9 & 34,6 \\
& $=$ ou > 8 anos & 17 & 65,4 \\
Período gestacional & Primeiro trimestre & 3 & 9,6 \\
& Segundo trimestre & 16 & 51,6 \\
& Terceiro trimestre & 12 & 38,7 \\
\hline N: número; (\%): Valores em percentual <: menor ; =: igual; >: maior. Boa Ventura de São Roque- \\
PR, 2014.
\end{tabular}

TABELA 3. ESTADO NUTRICIONAL PERÍODO PRÉ-GESTACIONAL E GESTACIONAL

\begin{tabular}{lccc}
\hline Estado nutricional & Pré-gestacional-\% (n) & Gestacional- \%(n) & $\mathbf{p}^{*}$ \\
\hline Baixo peso & $11,5(3)$ & $7,7(2)$ & \\
Eutrofia & $42,3(11)$ & $42,3(11)$ & 0,161 \\
Sobrepeso & $23,1(6)$ & $19,2(5)$ & \\
Obesidade & $23,1(6)$ & $30,8(8)$ & \\
\hline$\%)=$ Valores em percentual; $n=$ número; $\mathrm{p}^{*}$ = teste-t pareado. Boa Ventura de São Roque-PR, 2014.
\end{tabular}

A análise de comparação das variáveis presentes na sub-escala imagem corporal foi realizada entre ambos os períodos, sendo evidenciado diferença significante nas questões: "Sentiu-se atraente? " $(p=<0,001)$, "achou o seu rosto atraente?" $(p=0,018)$, "achou que seus seios estavam demasiadamente grandes?" ( $p=0,008)$ e "achou sua constituição física frágil ou débil?” ( $p=0,009)$. A maior média foi representada na questão “achou que seu corpo tinha um cheiro agradável?" tanto no período pré-gestacional como no gestacional $(p=0,203)$, sendo este item o que representou menor adequação de toda a sub escala imagem corporal. As questões que representaram médias maiores durante 0 período gestacional quando comparadas ao período pré-gestacional, foram as seguintes: "Achou que seus seios estavam demasiados grandes?" $(p=0,008)$, "Achou a sua constituição física frágil ou débil?" $(p=0,009)$ caracterizando uma pior percepção em relação 
a imagem corporal para estes itens durante a gestação (Tabela 4). "Achou que seus seios estavam demasiados grandes?" ( $p=0,008)$, "Achou a sua constituição física frágil ou débil?" $(p=0,009)$ caracterizando uma pior percepção em relação a imagem corporal para estes itens durante a gestação (Tabela 4).

TABELA 4. SUB ESCALA IMAGEM CORPORAL DO QUESTIONÁRIO MAMA, COMPARAÇÃO ENTRE PERÍODO PRÉ-GESTACIONAL E GESTACIONAL.

\begin{tabular}{|c|c|c|c|}
\hline \multirow[t]{2}{*}{ Descrição } & \multicolumn{3}{|l|}{$\begin{array}{c}\text { Pré- } \\
\text { gestacional }\end{array}$} \\
\hline & Média \pm DP & Média \pm DP & $\mathbf{p}^{*}$ \\
\hline Sentiu-se atraente $?^{*}$ & $2,88 \pm 0,71$ & $2,15 \pm 0,83$ & $<0,001$ \\
\hline Achou que seu corpo tinha um cheiro agradável?* & $2,96 \pm 0,72$ & $2,77 \pm 0,86$ & 0,203 \\
\hline Achou que seus seios estavam demasiado & $1,50 \pm 0,81$ & $1,42 \pm 0,57$ & 0,627 \\
\hline pequenos? & $2,73 \pm 0,82$ & $2,58 \pm 0,80$ & 0,425 \\
\hline Agradou-Ihe a forma do seu corpo?* & $2,69 \pm 0,67$ & $2,38 \pm 0,57$ & 0,018 \\
\hline Achou o seu rosto atraente?* & $1,62 \pm 0,98$ & $2,15 \pm 1,12$ & 0,008 \\
\hline Achou que seus seios estavam demasiados & $2,85 \pm 0,92$ & $2,73 \pm 0,82$ & 0,449 \\
\hline grandes? & $2,73 \pm 0,72$ & $2,50 \pm 0,76$ & 0,083 \\
\hline Sentiu orgulho da sua aparência física?* & $1,77 \pm 0,95$ & $1,73 \pm 0,87$ & 0,832 \\
\hline Sentiu o seu corpo suave e macio?* & $1,58 \pm 0,64$ & $2,0 \pm 0,84$ & 0,009 \\
\hline Sentiu o seu corpo desajeitado ou sem graça? & $2,42 \pm 0,90$ & $2,38 \pm 0,75$ & 0,832 \\
\hline Achou a sua constituição física frágil ou débil? & $1,85 \pm 1,04$ & $2,08 \pm 0,97$ & 0,247 \\
\hline Achou os seus seios atraentes?* & $24 \pm 5,85$ & $26,88 \pm 0,83$ & 0,005 \\
\hline \multicolumn{4}{|l|}{ Achou-se muito gorda? } \\
\hline Total sub-escala & & & \\
\hline
\end{tabular}

As correlações foram realizadas entre variáveis idade, estado civil, escolaridade, estado nutricional pré-gestacional, estado nutricional gestacional e semana gestacional, com a percepção da imagem corporal nos períodos pré-gestacional e gestacional. Foi encontrada uma correlação inversa significante $(p=0,024)$, porém regular $(0,442)$, entre escolaridade e percepção da imagem corporal no período gestacional.

\section{DISCUSSÃO}

O conhecimento de estado nutricional e percepção de imagem corporal são 
importantes para avaliar a coerência com o percebido e a realidade, principalmente no período gestacional, por ser uma fase de grandes modificações corporais.

Segundo a classificação do estado nutricional de acordo com o IMC prégestacional, o maior percentual 42,3\% $(n=11)$ é de eutrofia, porém, se somadas as demais classificações utilizadas, caracterizando estado nutricional inadequado das participantes com baixo peso, sobrepeso e obesidade, estas representam $57,7 \%(n=15)$ da amostra, 0 percentual de eutrofia foi inferior a encontrada em estudos como o de Melo et al., (2007), Nucci et al., (2001) e Rocha et al., (2005) onde o percentual mínimo de eutrofia correspondeu a $50 \%$.

Ao observar o estado nutricional da amostra no período gestacional mantém-se o percentual de eutrofia, sendo observado um crescimento no número de obesas $30,8 \%$ se comparado ao período anterior $23,1 \%$, o ganho de peso em excesso neste período foi observado também no estudo de Melo et al., (2007) e Rocha et al., (2005), podendo esse aumento, ser caracterizado devido a aumento de tecidos maternos, volume dos seios e crescimento do bebê. (ANDRETO et al., 2006).

O ganho excessivo de peso durante este período, representa risco de surgimento de doenças como diabetes mellitus gestacional e doença hipertensiva, e o ganho insuficiente de peso aumenta o risco de crianças nascidas com baixo peso (NUCCl et al., 2001; ROCHA et al., 2005). É importante que a mulher receba maior atenção no que diz respeito a orientação nutricional, principalmente nessa fase da vida, visto que nenhum desses extremos: ganho de peso em excesso e ganho de peso insuficiente, conduzem a resultados positivos.

Os resultados da sub-escala imagem corporal, mostraram que as mulheres apresentaram melhores respostas frente a esta sub-escala no período pré-gestacional, quando comparada ao período gestacional $24 \pm 5,85$ e $26,88 \pm 5,83$ respectivamente. Este resultado pode ser justificado pelas transformações que ocorrem no corpo da mulher neste período, no qual muitas vezes a mesma não está preparada o suficiente para receber estas mudanças. O estudo de Figueiredo et al., (2014) que avaliou gestantes adultas apresentou resultados ainda mais elevados $35,08 \pm 4,53$.

Das variáveis que apresentaram as maiores médias destacou-se a questão: "achou que seu corpo tinha um cheiro agradável?". Quando comparado ao estudo de Figueiredo et al. (2014), que apresentou média inferior nesta questão, evidencia-se uma percepção da imagem corporal menos favorável por parte das gestantes estudadas. As que apresentaram médias mais elevadas no estudo de Figueiredo et al., 2004 foram: "Achou que seus seios 
estavam atraentes?" e "Achou o seu rosto atraente?"”. Estes resultados podem ser encontrados devido às diferenças socioculturais visto que os estudos foram realizados com populações de diferentes países.

As questões que apresentaram médias superiores no período gestacional quando comparadas ao pré-gestacional foram: "Achou que seus seios estavam demasiados grandes?", "Achou a sua constituição física frágil ou débil?" caracterizando uma percepção da imagem corporal mais negativa em relação a estes itens no período gestacional, como já mencionado este período é característico de transformações, como o aumento no volume dos seios, e ganho de peso (COSTA et al., 2010), podendo ser esta a razão dos resultados encontrados visto que estas mudanças podem causar desconforto e até mesmo maior dificuldade de equilibrar o corpo, dificultando a pratica de atividades do cotidiano.

No estudo de Figueiredo et al., 2014, não houve significância na percepção da imagem corporal entre os indivíduos com escolaridade superior e inferior a 9 anos. No presente estudo a correlação apresenta que o maior tempo de escolaridade representa percepção da imagem corporal mais negativa no período gestacional. Em estudo realizado por Silva et al., (2012), com estudantes pode-se observar que a insatisfação da percepção da imagem corporal era maior em estudantes de mães com maior escolaridade.

Devido o local do estudo ser um município de pequeno porte, houve uma amostra bastante reduzida, a realização da pesquisa em dois momentos, inicial e final da gestação, poderiam mostrar resultados mais significativo, também são poucos os estudos validados relacionados a percepção da imagem corporal durante a gestação, dificultando a identificação de alterações.

\section{CONCLUSÃO}

O estado nutricional das participantes não teve modificações significantes entre os dois períodos, porém, apresentou maiores percentuais de obesidade no período gestacional, e a percepção da imagem corporal foi mais negativa durante a gestação. As informações apresentadas reforçam a importância da presença do nutricionista no acompanhamento de gestantes, apresentando orientações nutricionais, a fim de adequar o ganho de peso nesse período, melhorar a percepção da imagem corporal deste grupo, e reduzir o risco de desenvolvimento de enfermidades relacionadas a nutrição. 


\section{REFERÊNCIAS}

ANDRETO, L. M. et al. Fatores associados ao ganho ponderal excessivo em gestantes atendidas em um serviço público de pré-natal na cidade de Recife, Pernambuco, Brasil. Cadernos de Saúde Pública, v. 22, n. 11, p. 2401-2409, 2006. ISSN 0102-311X.

ATALAH SAMUR, E. et al. Propuesta de un nuevo estándar de evaluación nutricional en embarazadas. Rev. Med. Chile, v. 125, n. 12, p. 1429-36, 1997. ISSN 0034-9887.

BOSI, M. L. M. et al. Autopercepção da imagem corporal entre estudantes de nutrição: um estudo no município do Rio de Janeiro. J bras psiquiatr, v. 55, n. 2, p. 108-13, 2006.

BRASIL, M. Secretaria de Atenção à Saúde. Departamento de Ações Programáticas Estratégicas. Pré-natal e Puerpério: atenção qualificada e humanizada-manual técnico. Brasília: Ministério da Saúde, 2005.

COQUEIRO, R. D. S. et al. Insatisfação com a imagem corporal: avaliação comparativa da associação com estado nutricional em universitários. Rev. psiquiatr. Rio Gd. Sul, v. 30, n. 1, p. 31-38, 2008. ISSN 0101-8108.

COSTA, E. S.; PINON, G. M. B.; SOUSA, L. B. D. Alterações fisiológicas na percepção de mulheres durante a gestação. 2010. ISSN 1517-3852.

DREHMER, M. Ganho de peso gestacional, desfechos adversos da gravidez e retenção de peso pós-parto. 2010. Universidade Federal do Rio Grande do Sul

FIGUEIREDO, B.; MENDONÇA, M.; SOUSA, R. Versão portuguesa do maternal adjustment and maternal attitudes (MAMA). Psicologia, Saúde \& Doenças, p. 31-51, 2004. ISSN 1645-0086.

FIGUEIREDO, B.; TENDAIS, I.; DIAS, C. C. Maternal adjustment and maternal attitudes in adolescent and adult pregnant women. Journal of pediatric and adolescent gynecology, v. 27 , n. 4, p. 194-201, 2014. ISSN 1083-3188.

MELO, A. S. D. O. et al. Estado nutricional materno, ganho de peso gestacional e peso ao 
nascer. Revista Brasileira de Epidemiologia, v. 10, n. 2, p. 249-257, 2007. ISSN 1415-790X.

MENEZES, I. H. C. F.; DOMINGUES, M. H. M. D. S. Principais mudanças corporais percebidas por gestantes adolescentes assistidas em serviços públicos de saúde de Goiânia. Revista Nutrição, v. 17, n. 2, p. 185-194, 2004. ISSN 1415-5273.

NUCCI, L. B. et al. Nutritional status of pregnant women: prevalence and associated pregnancy outcomes. Revista de Saúde Pública, v. 35, n. 6, p. 502-507, 2001. ISSN 0034-8910.

OLIVEIRA, A. C. C. D.; SILVA, D.; PEREIRA, I. Impacto da escolaridade materna e paterna na perceção da imagem corporal em acadêmicos de Educação Física. 2012. ISSN 2182-2972.

ORGANIZATION, W. H. Obesity: preventing and managing the global epidemic. World Health Organization, 2000. ISBN 9241208945.

RAMOS, S. et al. O ajustamento materno na gravidez tardia: contributos para a prática de Enfermagem. Salucis Scientia-Revista de Ciências da Saúde da ESSCVP, v. 4, 2012.

ROCHA, D. D. S. et al. Estado nutricional e anemia ferropriva em gestantes: relação com o peso da criança ao nascer. Revista Nutrição, v. 18, n. 4, p. 481-489, 2005. ISSN 1415-5273.

ROMEIRO, A. A. F. Avaliação da implantação do Sistema de Vigilância Alimentar e Nutricional-SISVAN, no Brasil. 2010.

SAIKALI, C. J. et al. Imagem corporal nos transtornos alimentares. Revista de Psiquiatria Clínica, v. 31, n. 4, p. 164-166, 2004.

SIEGA-RIZ, A. M. et al. A systematic review of outcomes of maternal weight gain according to the Institute of Medicine recommendations: birthweight, fetal growth, and postpartum weight retention. American journal of obstetrics and gynecology, v. 201, n. 4, p. 339. e1-339. e14, 2009. ISSN 0002-9378.

SILVA, D. A. S.; PEREIRA, I. M. M.; OLIVEIRA, A. Impacto da escolaridade materna e paterna na perceção da imagem corporal em acadêmicos de Educação Física. Motricidade, v. 8, n. 2, p. 22-31, 2012. ISSN 1646-107X. 